\title{
Repairing Event Logs with Missing Events to Support Performance Analysis of Systems with Shared Resources
}

\author{
Vadim Denisov $^{1,3(\bowtie)}$, Dirk Fahland ${ }^{1}$, and Wil M. P. van der Aalst ${ }^{1,2}$ \\ ${ }^{1}$ Eindhoven University of Technology, Eindhoven, The Netherlands \\ \{v.denisov, d. fahland\}@tue.nl \\ 2 Process and Data Science (Informatik 9), RWTH Aachen University, Aachen, Germany \\ wvdaalst@pads.rwth-aachen.de \\ 3 Vanderlande Industries, Veghel, The Netherlands
}

\begin{abstract}
To identify the causes of performance problems or to predict process behavior, it is essential to have correct and complete event data. This is particularly important for distributed systems with shared resources, e.g., one case can block another case competing for the same machine, leading to inter-case dependencies in performance. However, due to a variety of reasons, real-life systems often record only a subset of all events taking place. For example, to reduce costs, the number of sensors is minimized or parts of the system are not connected. To understand and analyze the behavior of processes with shared resources, we aim to reconstruct bounds for timestamps of events that must have happened but were not recorded. We present a novel approach that decomposes system runs into token trajectories of cases and resources that may need to synchronize in the presence of many-to-many relationships. Such relationships occur, for example, in warehouses where packages for $\mathrm{N}$ incoming orders are not handled in a single delivery but in $\mathrm{M}$ different deliveries. We use linear programming over token trajectories to derive the timestamps of unobserved events in an efficient manner. This helps to complete the event logs and facilitates analysis. We focus on material handling systems like baggage handling systems in airports to illustrate our approach. However, the approach can be applied to other settings where recording is incomplete. The ideas have been implemented in ProM and were evaluated using both synthetic and real-life event logs.
\end{abstract}

Keywords: Log repair $\cdot$ Process mining $\cdot$ Performance analysis $\cdot$ Modeling $\cdot$ Material handling systems

\section{Introduction}

Precise knowledge about actual process behavior and performance is required for identifying causes of performance issues [16], as well as for predictive process monitoring of important process performance indicators [14]. For Material Handling Systems (MHS), such as Baggage Handling Systems (BHS) of airports, performance incidents are usually investigated offline, using recorded event data for finding root causes of 
problems [10], while online event streams are used as input for predictive performance models [4]. Both analysis and monitoring heavily rely on the completeness and accuracy of input data. For example, events may not be recorded and, as a result, we do not know when they happened even though we can derive that they must have happened. Yet, when different cases are competing for shared resources, it is important to reconstruct the ordering of events and provide bounds for non-observed timestamps.

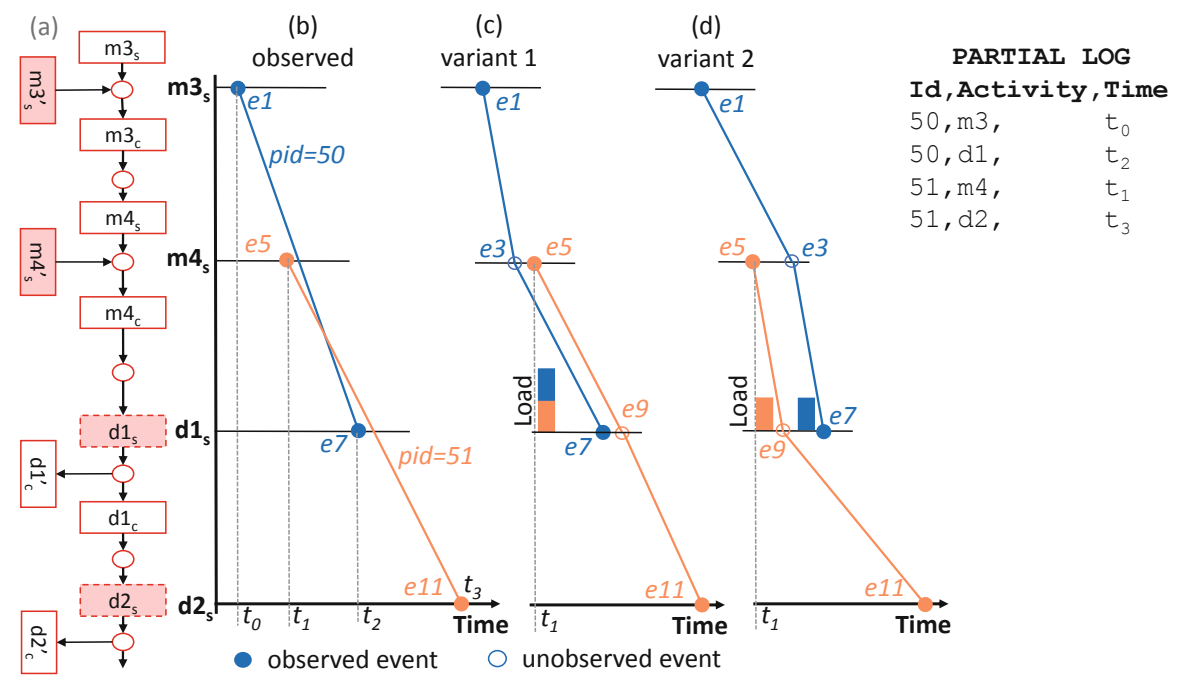

Fig. 1. An MHS model example (a), observed imprecise behavior for two cases 50 and 51 (b), possible actual behaviors (c,d).

However, in most real-life systems, items are not continuously tracked and not all events are stored for cost-efficiency, leading to incomplete performance information which impedes precise analysis. For example, an MHS tracks the location of an item, e.g., a bag or box, via hardware sensors placed throughout the system, generating tracking events for system control, monitoring, analysis, and prediction. Historically, to reduce costs, a tracking sensor is only installed when it is strictly necessary for the correct execution of a particular operation, e.g., only for the precise positioning immediately before shifting a bag from one conveyor onto another. Moreover, even when a sensor is installed, an event still can be discarded to save storage space. As a result, the recorded event data of an MHS are typically incomplete, hampering analysis based on such incomplete data. Therefore, it is essential to repair the event data before analysis. Figure 1 shows a simple MHS where events are not always recorded. The process model is given and for two cases the recorded incomplete sets of events are depicted using the so-called Performance Spectrum [10].

Figure 1(b) shows item pid $=50$ entering the system via $m 3$ at time $t_{0}$ (event $e_{1}$ ) and leaving the system via $d 1$ at time $t_{2}\left(e_{7}\right)$, and item pid $=51$ entering the system via $m 4$ at time $t_{1}\left(e_{5}\right)$ and leaving the system via $d 2$ at time $t_{3}\left(e_{11}\right)$. As only these four events 
are recorded, the event data do not provide information in which order both cases traversed the segment $m 4 \rightarrow d 1$. Naively interpolating the movement of both items, as shown in Fig. 1(b), suggests that item pid $=51$ overtakes item pid $=50$. This contradicts that all items are moved from $m 4$ to $d 1$ via a conveyor belt, i.e., a FIFO queue: item 51 cannot have overtaken item 50. In contrast, Fig. 1(c) and Fig. 1(d) show two possible behaviors that are consistent with our knowledge of the system. We know that a conveyor belt (FIFO queue) is a shared resource between $m 4$ and $d 1$. Both variants differ in the order in which items 50 and 51 enter and leave the shared resource, the speed with which the resource operated, and the load and free capacity the resource had during this time. In general, the longer the duration of naively interpolated segment occurrences, the larger the potential error. Errors in load, for example, make performance outlier analysis [10] or short-term performance prediction [9] rather difficult. Errors in order impede root-cause analysis of performance outliers, e.g., finding the cases that caused or were affected by outlier behavior.

Problem. In this paper, we address a novel type of problem as illustrated in Fig. 1 and explained above. The behavior and performance of the system cannot be determined by the properties of each case in isolation, but depends on the behavior of other cases and the behavior of the shared resources involved in the cases. Crucially, each case is handled by multiple resources and each resource handles multiple cases, resulting in many-to-many relations between them. The concrete problem we address is to reconstruct unobserved behavior and performance information of each case and each shared resource in the system that is consistent with both observed and reconstructed unobserved behavior and performance of all other cases and shared resources. More specifically, we consider the following information as given: (1) an event $\log L_{1}$ containing the case identifier, activity and time for recorded events where intermediate steps are not recorded (i.e., the event log may be incomplete), (2) a model of the process (i.e., possible paths for handling each individual case), and (3) a description (model) of the resources involved in each step (e.g., queues, single server resources and their performance parameters such as processing and waiting time). Based on the above input, we want to provide a complete event $\log L_{2}$ that describes (1) for each case the exact sequence of process steps, (2) and for each unobserved event a time-window of earliest and latest occurrence of the event so that (3) either all earliest or all latest timestamps altogether describe a consistent execution of the entire process over all shared resources.

Contribution. We propose a solution to this problem for a limited class of systems. We focus on processes where each step is served by one single-server resource and resources are connected by strict FIFO queues only. These assumptions are reasonable for a large class of MHSs. Our current solution formulation assumes the process to be acyclic which suffices for many real-life problem instances. Section 2 presents related work while elaborating on the problem. Specifically, prior work either only considers the case or the resource perspective explicitly, making implicit assumptions about their complex interplay. To overcome this limitation, we use synchronous proclets [11] in Sect. 3 to conceptually decompose a run of a system into individual token trajectories of cases, resources, and queues. Token trajectories synchronize when a resource or queue is involved in a case, allowing to explicitly describe their many-to-many relations in the run. Section 4 then formally captures token trajectories in terms of partial orders of 
events and defines the general problem. We solve the problem in Sect. 5 by formulating a Linear Programming (LP) problem [19] in terms of timestamps along the different token trajectories. To evaluate the approach, we compare the restored event logs with the ground truth for synthetic logs and estimate errors for real-life event logs for which the ground truth is unavailable (Sect.6). We discuss our findings and future work in Sect. 7.

\section{Related Work}

In all operational processes (logistics, manufacturing, healthcare, education and so on) complete and precise event data, including information about workload and resource utilization, is highly valuable since it allows for process mining techniques uncovering compliance and performance problems. Event data can be used to replay processes on top of process models [2], to predict process behavior [5,9], or to visualize detailed process behavior using performance spectra [10]. All of these techniques rely on complete and correct event data. Since this is often not the case, we aim to transform incomplete event data into complete event data.

Various approaches exist for dealing with incomplete data of processes with nonisolated cases that compete for scarce resources. In call-center processes, thoroughly studied in [12], queueing theory models can be used for load predictions under assumptions about distributions of unobserved parameters, such as customer patience duration [6], while assuming high load snapshot principle predictors show better accuracy [21]. For time predictions in congested systems, the required features are extracted using congestion graphs [20] mined using queuing theory.

Techniques to repair, clean, and restore event data before analysis have been suggested in other works. An extensive taxonomy of quality issue patterns in event logs is presented in [22]. The taxonomy also lists approaches to repair inadvertent time intervals [22] in [8]. In [15] resource availability calendars are retrieved from event logs without the use of a process model, but assuming start and complete life-cycle transitions as well as a case arrival time present in a log. Using a process model, classical trace alignment algorithms [7] restore missing events but do not restore their timestamps. The authors conclude (see [7], p. 262) that incorporating other dimensions, e.g., resources, for multi-perspective trace alignment and conformance checking is an important challenge for the near future. Recently, also techniques for process discovery and conformance checking over uncertain event data were presented [17,18]. The output of our approach can provide the input needed for these techniques.

Our work contributes to the problem of reconstructing behavior of cases and limited shared resources for which the cases compete. We use the notion of proclets first introduced in [1] and adapted for process mining in [11] to approach the problem from control-flow and resource perspectives at once. We assume a system model given as a composition of a control-flow proclet (process) and resource/queue proclets. We restore missing events through classical trace alignments over control-flow proclets. The dynamic synchronization of proclets [11] allows us to infer how and when resource tokens must have traversed over the control-flow steps, which we express as a linear programming problem to compute timestamp intervals for the restored events. Event logs repaired in this way enable the use of analysis assuming complete event logs. 


\section{Modeling Inter-Case Behavior via Shared Resources}

Prior work (cf. Sect. 2) approaches the problem of analyzing the performance of systems with shared resources primarily either from the control-flow perspective $[5,9,15,17,18]$ or the resource/queuing perspective [6,12,20,21], leading to information loss about the other perspective. In the following, we show how to conceptualize the problem from both perspectives at once using synchronous proclets [11]. This way we are able to capture both control-flow and resource dynamics and their interaction as synchronizing token trajectories. We introduce the model in Sect. 3.1 and use it to illustrate how incomplete logging incurs information loss for performance analysis in Sect. 3.2.
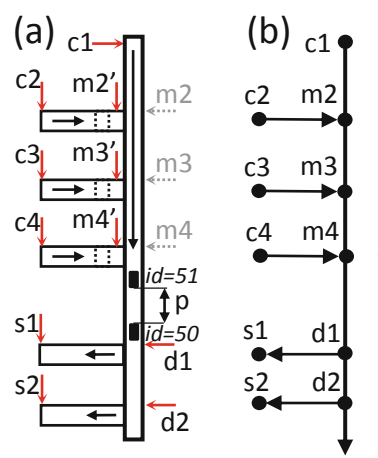

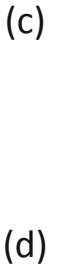

Event, Id, Activity, Time

e0, 50,c3,01.01.20 9:00:15

$\mathrm{e}, \mathrm{s}, \mathrm{m} 3,01.01 .209: 00: 30$

$e 3,50, m 4,01.01 .20$ 9:00:45

e7, 50,d1,01.01.20 9:01:00 e18,50,s1,01.01.20 9:01:15

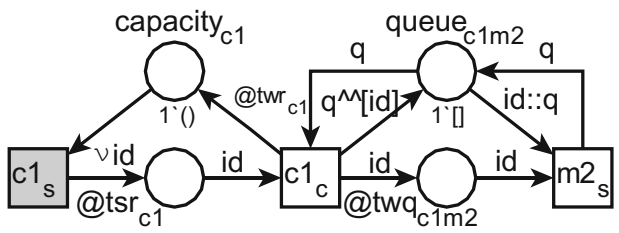

PARTIAL LOG 1

Event, Id, Activity, Time e17,51,c4,01.01.20 9:00:35 e5, 51,m4,01.01.20 9:00:50 e9, 51,d1,01.01.20 9:00:05 e11,51,d2,01.01.20 9:01:20 e19,51,s2,01.01.20 9:01:35

Fig. 2. A baggage handling system fragment (a) and its material flow diagram (b). Conveyor belts of check-in counters $c 1-c 4$ merge at points $m 2-m 4$, further downstream bags can divert at $d 1$ and $d 2$ to X-Ray security scanners $s 1$ and $s 2$. Red arrows show sensor (logging) locations. Conveyor $c 1: m 2$ is modeled as a coloured Petri net model (c). An example of an incomplete event log of the system in (a) is shown in (d), where missing events are shown in the grey color. (Color figure online)

\subsection{Processes-Aware Systems with Shared Resources}

We explain the dynamics of process-aware systems over shared resources using a BHS handling luggage. The process control-flow takes a bag from a source (e.g., check-in or transfer from another flight), to a destination (e.g., the airplane, transfer) along intermediate process steps (e.g., baggage scanning, storage). BHS resources are primarily single-server machines (e.g., baggage scanners) connected via conveyor belts, i.e., FIFO queues. Figure 2(a) shows a typical system design pattern involving the control-flow and resource perspective: four parallel check-in desks (c1-c4) merge into one linear conveyor through merge points (m2-m4). Divert points (d1 and d2) can route bags from the linear conveyor to scanners (s1 and s2). Each merge point and scanner is preceded by a FIFO queue for buffering incoming cases (bags) in case the corresponding resource is busy. Figure 2(b) shows the plain control-flow of this BHS (also called Material Flow 
Diagram (MFD)). A real-life BHS may contain hundreds of process steps and resources, and conveyors may also form loops.

Modeling with Coloured Petri Nets. Figure 2(c) shows a Coloured Petri Net (CPN) model for the segment $c 1 \rightarrow m 2$. In the model, transitions $c 1_{s}$ and $c 1_{c}$ describe start and completion of the check-in step $c 1$. At the occurrence of $c 1_{s}$ a new bag $(v i d)$ represented by a token with an id is inserted. Step $c 1$ is served by a single resource (place capacity ${ }_{c 1}$ ) which has service-time $t s r_{c 1}$ to complete the step and waiting time $t r w_{c 1}$ until the next bag can go through $c 1$. All Resources in a BHS may require a waiting time to ensure sufficient "operating space" between two subsequent cases. After completion of $c 1$, the bag enters a FIFO queue (modeling a conveyor belt) to the start of the merge step $m 2_{s}$. Time annotation $t w q_{c 1 m 2}$ models the minimum time it takes for a bag to travel from $c 1$ to $m 2$. Only then a bag may leave the queue at $m 2_{s}$. The CPN model in Fig. 2(c) describes the impact of limited resource capacity and queues on the progress of a case, but does not model the resource itself as its own entity. The absence of the resource in the described behavior makes it impossible to reason about its behavior explicitly.

Modeling with Synchronous Proclets. The synchronous proclet system in Fig. 3 describes the entire BHS of Fig. 2(a) by using three types of proclets.

1. The process proclet (red border) is a Petri net describing the control-flow perspective of how bags may move through the system. It directly corresponds to the MFD of Fig. 2(b). It is transition-bordered and each occurrence of one of its initial transitions creates a new case identifier, see [11] for details.

2. Each resource proclet (green border) models a resource as its own entity with a cyclic behavior. For example, the PassengerToSystemHandover proclet (top left) identifies a concrete resource by token id $c 1$; its life-cycle models that starting a task $\left(c 1_{s}\right)$ makes the resource busy and takes service time $t s r_{c 1}$, after completing the task $\left(c 1_{c}\right)$ the resource has waiting time $t w r_{c 1}$ before being idle again in the same way as Fig. 2(c). All other resource proclets follow the same pattern, though some resources such as MergingUnit-m2 and DivertingUnit-dl may have two transitions to become busy or idle, respectively.

3. Each queue proclet (blue border) describes a FIFO queue as in Fig. 2(c). However, the queue state (the list) is accompanied by a queue identifier in place $q$. Items entering the queue are remembered by their number (generated from the count place).

The proclet system synchronizes process, resources, and queues via synchronous channels between transitions. Transitions linked via synchronous channels may only occur when all linked transitions are enabled; when they occur, they occur in a single synchronized event. For example, transition $c 1_{s}$ is always enabled in Process, generating a new bag id, e.g., id $=49$, but it may only occur together with $c 1_{s}$ in PassengerToSystemHandover, i.e., when resource $c 1$ is idle, thereby synchronizing the process case for bag $i d=49$ with the resource with identifier $c 1$. By annotation init $c 1,1: 1 c 1$ is now correlated to $i d=49$. The subsequent correlation annotation $=c 1,1: 1$ on the channel of the complete transition $c 1_{c}$ ensures that resource $c 1$ only synchronizes with the process case on which it started the step, i.e., id $=49$; the next occurrence of $c 1_{s}$ will create a new correlation to another process case, see [11] for details. In the example, each resource is statically linked to one process step, but the model also allows for one 


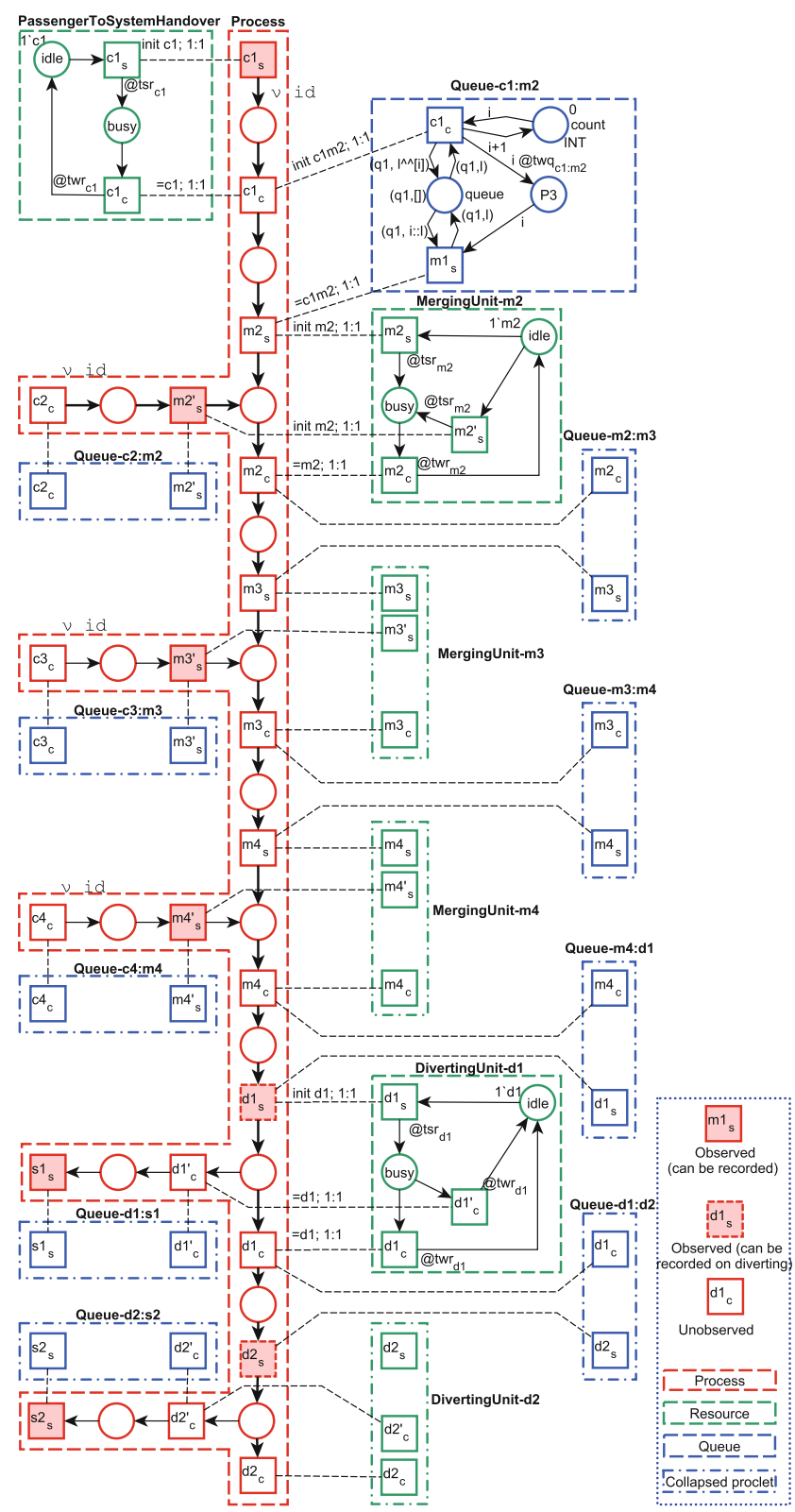

Fig. 3. The synchronous proclet model of the system shown in Fig. 2(a) consists of three types of proclets: Process for modeling a system layout and process control flow (red), Resource for modeling connector and sensor resources (services), and Queue for modeling conveyors transporting bags in the FIFO order. Only filled transitions can be observed in an event log. (Color figure online) 


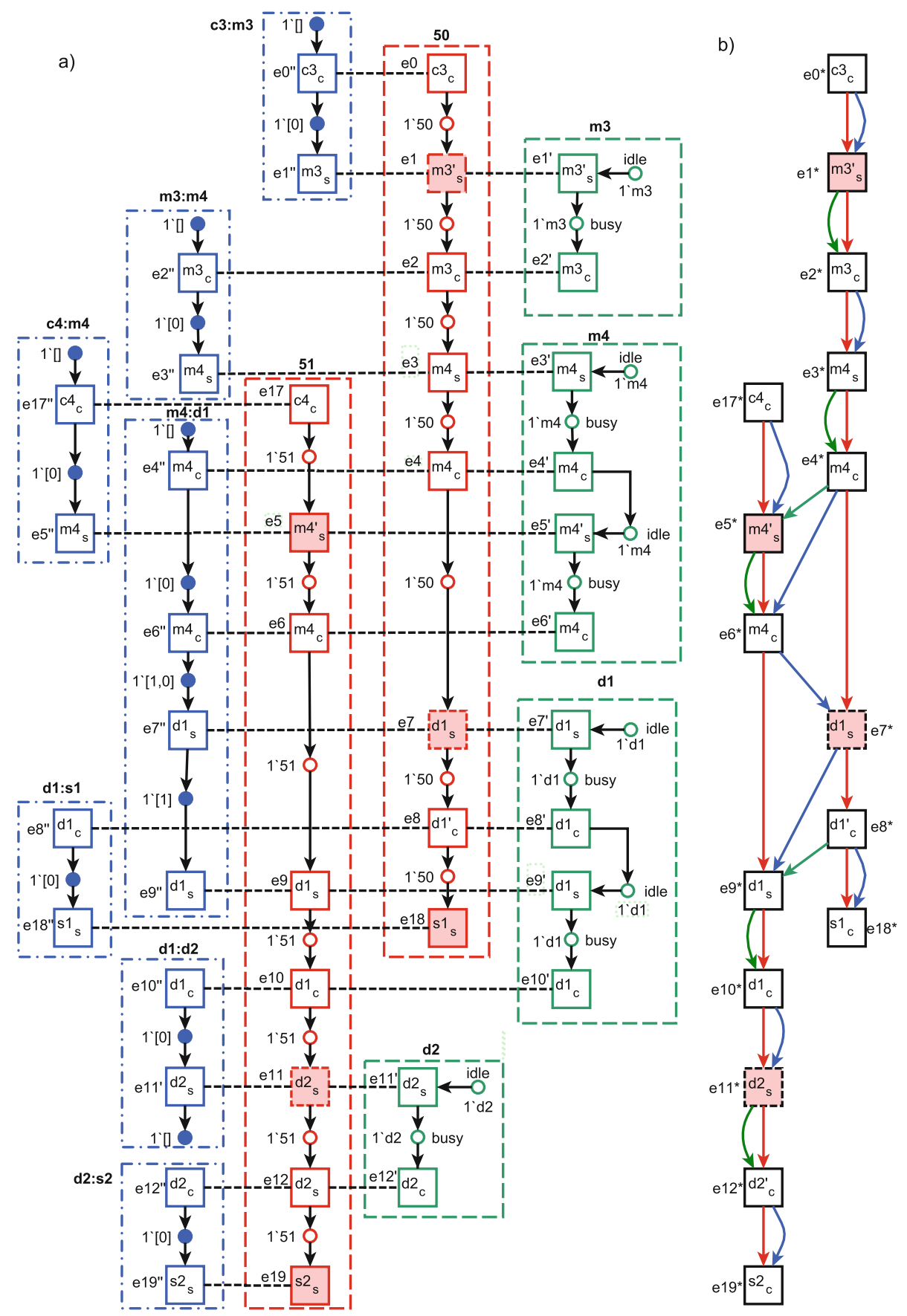

Fig. 4. Synchronization of multiple sub-runs of the synchronous proclet system in Fig. 3 over shared resources and queues (a), and a global partial order obtained by the union of partial orders of each sub-run (b) for synchronized events, shown by red, green and blue arrows for partial orders $<_{\text {pid }},<_{\text {rid }}$ and $<_{\text {qid }}$ respectively. (Color figure online) 
resource to participate in multiple different process steps, and multiple resources to be required for one process step. In the following, we call a proclet system that defines proclets for processes, queues, and resource that are linked via synchronous channels as described above, a $P Q R$ system.

Proclets Describe Synchronizing Token Trajectories. We now highlight how the partial-order semantics of synchronous proclets [11] preserves the identities of process, resources, and queues as "token trajectories". Figure 4(b) shows a partially-ordered run of the PQR system of Fig. 3 for two bags $i d=50$ and $i d=51$. The run in Fig. 4(b) can be understood as a synchronization of multiple runs of the process, resource, and queue proclets, one for each case, resource, or queue involved as shown in Fig. 4(a).

Bag 50 gets inserted via input transition $c 3_{c}$ (event $e_{0}^{*}$ in Fig. 4(b)). This event is a synchronization of events $e 0$ ( $c 3_{c}$ occurs for bag 50 in the Process proclet) and $e 0^{\prime}\left(c 3_{c}\right.$ occurs for the $c 3: m 3$ queue) in Fig. 4(a). The minimal waiting time $t w q_{c 3 m 3}$ must pass before bag 50 reaches the end of the queue and process step $m 3$ can start. The process step $m 3$ merges bag 50 from the check-in conveyor $c 3$ onto the main linear conveyor and may only start via transition $m 3_{s}$ when MergingUnit-m3 is idle. As this is the case, bag 50 leaves the queue ( $e 1^{\prime \prime}$ in $\left.c 3: m 3\right), m 3$ starts merging ( $e 1^{\prime}$ in $m 3$ ), the bag starts the merging step (event $e 1$ in Process), resulting in the synchronized event $e 1^{*}$ in Fig. 4(b).

By $e_{1}^{\prime}$, resource $m 3$ switches from idle to busy and takes time $t s r_{m 3}$ before it can complete the merge step with $m 3_{c}$ (event $e 2^{\prime}$ ) on bag 50 (event $e 2$ ); this merge step also inserts bag 50 into queue $m 3: m 4\left(e 2^{\prime \prime}\right)$ resulting in synchronized event $e 2^{*}$. Subsequently, bag 50 leaves queue $m 3: m 4\left(e 3^{*}\right)$ is pushed by merge unit $m 4$ into queue $m 4: d 1$ $\left(e 4^{*}\right)$.

Concurrently, bag 51 is inserted via input transition $c 4_{c}$ (event $e 17^{*}$ ), moves via queue $c 4: m 4$ also to merge unit $m 4$ to enter queue $m 4: d 1$, i.e., both bags 50 and 51 now compete for merge unit $m 4$ and the order of entering $m 4: d 1$. In the run in Fig. 4 , $m 4$ executes $m 4_{s}$ and $m 4_{c}$ for bag $51\left(e 5^{*}\right.$ and $\left.e 6^{*}\right)$ after completing this step for bag $50\left(e 3^{*}\right.$ and $\left.e 4^{*}\right)$. Thus, 51 enters the queue $\left(e 6^{*}\right)$ after 50 entered the queue $\left(e 5^{*}\right)$ but before 50 leaves the queue $e 7^{*}$. Consequently, divert unit $d 1$ first serves $50\left(e 7^{*}\right.$ and $\left.e 8^{*}\right)$ to reach scanner $s 1\left(e 18^{*}\right)$ before serving $51\left(e 9^{*}\right.$ and $\left.e 10^{*}\right)$ to reach scanner $s 2\left(e 19^{*}\right)$.

Figure 4(b) shows how the process tokens of bag 50 and 51 synchronized with the resources and queue tokens along the run, forming sequences or trajectories of events where this token was involved. For example, bag 50 followed the trajectory $e 0^{*}, e 1^{*}, \ldots, e 8^{*}, e 18^{*}$ and queue $m 4: d 1$ followed trajectory $e 4^{*}, e 6^{*}, e 7^{*}, e 9^{*}$ thereby synchronizing with both bag 50 and bag 51 .

\subsection{Information Loss Because of Incomplete Logging}

Although event data on objects that are tracked can be used for various kinds of data analysis [4,9], in practice sensors are placed only where it is absolutely necessary for correct operation of the system, e.g., for merge and divert operations, without considering data analysis needs. Applied to our example, only the transitions that are shaded in Fig. 3 would be logged, i.e., $c 1_{s}, m 2_{s}^{\prime}, m 3_{s}^{\prime}, m 4_{s}^{\prime}, d 1_{s}, d 2_{s}, s 1_{s}, s 2_{2}$ would be logged from the control-flow perspective only. The run of Fig. 4 would result in a "typical" but highly incomplete event log as shown in Fig. 2(d). 
According to this incomplete log, bag 50 silently passes $m 4$ and is tracked again only at $d l(e 7)$ and finally at $s l(e 18)$ whereas 51 silently passes $d l$ (as it moves further on the main conveyor) and is tracked again only at $d 2(e 11)$. Based on this incomplete information the bags 50 and 51 may have traversed $m 4: d 1$ in different orders and at different speeds resulting also in different loads as illustrated in Fig. 1. As a result, in case of congestion, we cannot determine the ordering of cases [10], cannot compute the exact load on each conveyor part for (predictive) process monitoring [5,9]. The longer an unobserved path (e.g., $c 1 \rightarrow d 2$ ), the higher the uncertainty about the actual behavior and the less accurate performance analysis outcome.

Although minimal (or even average) service and waiting times on conveyor belts and resource are known, we need to determine the exact timestamps of all missing events and their order to reconstruct for how long resources were occupied by particular cases and in which order cases were handled, e.g., did 50 precede 51 on $m 4: d l$ or vice versa?

The objective of this paper is to reconstruct from a subset of events logged from the control-flow perspective only the remaining events (including time information), so that the time order is consistent with a partially ordered run of the entire system, including resource and queue proclets. For example, from the recorded events of the event log in Fig. 2(d) we reconstruct the remaining events (Fig. 4(a)) with time information so that the resulting order (by time) is consistent with the partially ordered run in Fig. 4(b).

\section{System Runs and Partial Event Logs}

In Sect. 3, we showed how the behavior of resource and queue-aware processes can be modeled as a PQR system, a particular type of a synchronous proclet system. The partially-ordered run of a PQR system decomposes into token trajectories for process cases, resources, and queues. In this section, we first formalize this relation between a partially ordered run of a system and its token trajectories through projection on partially ordered sets. We then formalize partial and complete event logs of a system run within this model and state the formal problem we address.

We use the following notion. Let $A$ be a set of event classifiers; $A$ is usually the set of activity names or the set of locations in case of an MHS. Let $T$ be the set of time durations and timestamps, e.g., the rational or real numbers. Let $\mathcal{E}$ be the universe of unique events with attributes, let $A N$ be a set of attribute names. For any $e \in \mathcal{E}, n \in A N$, $\#_{n}(e)$ is the value of attribute $n$ for event $e\left(\#_{n}(e)=\perp\right.$ if attribute $n$ is undefined for $\left.e\right)$. Each event has a mandatory attribute $a c t, \#_{a c t}(e) \in A$, a mandatory attribute $l t$ for a lifecycle transition, $\#_{l t}(e) \in\{$ start, complete $\}$ and an optional attribute time, $\#_{\text {time }}(e) \in T$. Finally, we allow events to be related to multiple case notions. Let $\mathcal{Z}$ be the universe of case identifiers and $I D \subset A N$ be a set of case notions. If $\#_{i d}(e)=z$, then event $e$ is related to case $z$ under case notion $i d \in I D$.

From Partial Orders to Token Trajectories. A run of a proclet system [11] can be observed in terms of a Strict Partially Ordered Set (SPOSET) $\pi=(E,<)$ of events $E \subseteq \mathcal{E}$. As usual, we write $e_{1}<e_{2}$ if event $e_{1}$ precedes event $e_{2}$ and we write $e_{1} \lessdot e_{2}$ iff $e_{1}$ directly precedes $e_{2}$, i.e., $e_{1}<e_{2}$ and there is no other event $e_{3}$ with $e_{1}<e_{3}<e_{2}$. In a $\mathrm{PQR}$ system we can distinguish three case notions $I D=\{$ pid, rid, qid $\}$ to distinguish 
cases of the process, resources, and queues. Each event $e \in \mathcal{E}$ in a run of a PQR system has one or more case notions from $I D$. For example, in Fig. $4, \#_{\text {pid }}\left(e 5^{*}\right)=51, \#_{\text {rid }}\left(e 5^{*}\right)=$ $m 4, \#_{\text {qid }}\left(e 5^{*}\right)=c 4: m 4$.

Restricting $<$ of a system run to events of the same case notion $i d \in I D$ results in a case notion-specific partial order $e_{1}<i d e_{2}$ iff $e_{1}<e_{2}$ and $\#_{i d}\left(e_{1}\right)=\#_{i d}\left(e_{2}\right) \neq \perp$. Only events which share the same case notion and case identifier are ordered by $<_{i d}-$ events of different cases are unordered. For example, in Fig. 4(b) $e 4^{*}<_{\text {rid }} e 5^{*}$ but $e 4^{*} \nless_{\text {pid }} e 5^{*}$. Consequently, $<_{\text {pid }}$ orders all events wrt. the process perspective whereas $<_{\text {rid }}$ and $<_{\text {qid }}$ order all events wrt. the resource and queue perspective, respectively. For a given case notion $i d \in I D$ and case identifier $z \in \mathcal{Z}$, the events $E_{i d}^{z}=\left\{e \in E \mid \#_{i d}(e)=z\right\}$ of case $z$ and Strict Partial Order (SPO) $<\left._{i d}^{z}\right|_{E_{i d}^{z} \times E_{i d}^{z}}$, restricted to the events of the same case, define a sub-run $\pi_{i d}^{z}=\left(E_{i d}^{z},<_{i d}^{z}\right)$. Each such sub-run formalizes one token trajectory in the system run $\pi$. For example, Fig. 4(a) shows the sub-runs, viz. token trajectories, of all cases of the run of Fig. 4(b), i.e., $\pi_{p i d}^{50}$ and $\pi_{p i d}^{51}$ from the perspective of the process, $\pi_{\text {rid }}^{m 3}, \pi_{\text {rid }}^{m 4}, \pi_{\text {rid }}^{d 1}, \pi_{\text {rid }}^{d 2}$ from the perspective of the resources, and $\pi_{\text {qid }}^{c 3: m 3}, \pi_{\text {qid }}^{m 3: m 4}, \pi_{\text {qid }}^{c 4: m 4}, \pi_{\text {qid }}^{m 4: d 1}, \pi_{\text {qid }}^{d 1: d 2}, \pi_{\text {qid }}^{d 1: s 1}, \pi_{\text {qid }}^{d 2: s 2}$ from the perspective of the queues. In this way, our model shows that events of different process cases ( $p i d=50$ and pid $=51$ ) are independent under the classical control-flow perspective $<_{\text {pid }}$, e.g., $e 4^{*} \nless_{\text {pid }} e 5^{*} \nless_{\text {pid }} e 7^{*}$, but mutually depend on each other under $<_{\text {rid }}$ and $<_{\text {qid }}$, e.g., $e 4^{*}<_{\text {rid }} e 5^{*}<_{\text {rid }} e 6^{*}$ and $e 6^{*}<_{q i d} e 7^{*}$. Each sub-run $\pi_{i d}^{z}$ is a "proper" run of case $z$ in the corresponding proclet (Lemma 2 in [11]).

Event Logs and Token Trajectories. Starting point for our analysis is the notion of a classical control-flow event log, which we express in our model of SPOSETs using pid as case notion. An event $\log L=(E,<)$ is a finite set of events $E$ where each event $e$ has an activity $\#_{a c t}(e)$, a process case id $\#_{p i d}(e)$. Note that $e$ may have additional case identifiers $\#_{\text {rid }}(e)$ and $\#_{\text {qid }}(e)$ as attributes.

Adopting [13] to our setting, the optional timestamps $\#_{\text {time }}(e)$ induce the log's partial order, i.e., two events $e_{1}$ and $e_{2}$ are ordered if $e_{1}$ time-wise precedes $e_{2}$ and both are related in some case (for any $i d \in I D$ ), i.e., $e_{1}<e_{2}$ iff $\perp \neq \#_{\text {time }}\left(e_{1}\right)<\#_{\text {time }}\left(e_{2}\right) \neq \perp$ and there exists $i d \in I D$ with $\#_{i d}\left(e_{1}\right)=\#_{i d}\left(e_{2}\right)$. If all events are only related to $i d$ cases, then $<$ and $<_{i d}$ are identical. Further, each sub-run $L_{i d}^{z}$ (projection onto events with $\#_{i d}(e)=z$ ) is a trace for case $z$ under case notion $i d$.

Event Logs and Token Trajectories. Given a model $M$ of a PQR system, i.e., a system defining proclets for pid, rid, qid, we call $\log L$ complete wrt. events and ordering iff there is a run $\pi$ of $M$ such that $L$ and $M$ are isomorphic wrt. attributes act, pid, qid, rid. Note that the ordering in $L$ is induced by event timestamps only, thus a complete log defines the "right" timestamps. Further note that in a complete $\log L$, each trace $L_{i d}^{z}$ is also complete and describes a token trajectory, i.e., a sub-run $\pi_{i d}^{z}$ of $\pi$ that fits the corresponding proclet. Further, all traces of process cases ( $p i d$ ) are ordered relative to each other via the shared resources and queues as described in $M$.

In reality often only a subset of activities $B \subseteq A$ and the control-flow case notion pid have been recorded in a log, making it partial. In this paper, we call a $\log L^{\prime}$ partial if there exists a complete $\log L=(E,<)$ of $M$ (viz. system run $\pi$ ) such that $L^{\prime}=\left(E^{\prime},<^{\prime}\right)$ is 
the projection of $L$ onto activities in $B,\left.L\right|_{B}=\left(E_{B},<\left.\right|_{E_{B} \times E_{B}}\right), E_{B}=\left\{e \in E \mid \#_{\text {act }}(e) \in B\right\}$ such that additionally

1. each $e \in E^{\prime}$ has only case notion pid, i.e., $\#_{\text {pid }}(e) \neq \perp, \#_{\text {rid }}(e)=\#_{\text {qid }}(e)=\perp$,

2. $\#_{\text {time }}(e)$ is defined, and

3. for each process case $z$ occurring in $L, L^{\prime}$ contains at least the first and last event of the complete trace.

Thus, $L^{\prime}$ contains for each case $z$ at least one partial trace $L_{\text {pid }}^{\prime z}$ recording the entry and exit of the case and preserving the order of observed events, i.e., it can be completed to fit the model. An MHS typically records a partial log as defined above. Figure 2(d) shows a partial event log of the run on Fig. 4. In a partial event log, events of different process cases are less ordered, e.g., observed events $e 1^{*}$ and $e 5^{*}$ in Fig. 4 are unordered wrt. any resource or queue whereas they are ordered in the complete run. In the following, we investigate how to restore this lost ordering.

Problem Formulation. Reconstructing a complete log from a partial log as defined above requires to reconstruct all missing events, all missing case notion attributes, and their timestamp. Restoring the exact timestamp is generally infeasible and for most use cases also not required. We, therefore, formulate the problem as restoring time-windows providing minimal and maximal timestamps for each unobserved event.

Let $M$ be a model of a PQR system defining life-cycles of process, resource, and queue proclets, which resources and queues synchronize on which process step, and for each resource the minimum service time $t s r$ and waiting time $t w r$ and for each queue the minimum waiting time $t w q$. Given $M$ and a partial $\log L_{1}=\left(E_{1},<_{1}\right)$ of $M$, we want to (1) reconstruct unobserved events $E_{u}$ for all process cases in $L_{1}$ and their relations to queues and resources, and (2) for each unobserved event $e \in E_{u}$ a timewindow of earliest and latest occurrence of the event $\#_{\text {tmin }}(e), \#_{\text {tmax }}(e) \in T$ so that (3) $L_{2}=\left(E_{1} \cup E_{u},<_{2}\right)$ is a complete $\log$ of $M$ when $<_{2}$ is inferred from $\#_{\text {tmin }}(e)$ or from $\#_{\text {tmax }}(e)$.

\section{$5 \quad$ Inferring Timestamps Along Token Trajectories}

In Sect. 4, we presented the problem of restoring missing events and time-windows for their timestamps from a partial event $\log L_{1}$ such that the resulting $\log$ is consistent with resource and queueing behavior. In this section, we solve the problem for PQR systems with acyclic process proclets by casting it into a constraint satisfaction problem, that can be solved using Linear Programming (LP) [19]. In Sect. 5.1, we show how to infer unobserved events (from $M$ ) and how to infer resource and queue identifiers from $M$ to construct an intermediate $\operatorname{SPO}\left(E_{2},<_{2}\right)$. All unobserved events $E_{2} \backslash E_{1}$ have no timestamp, i.e., they are unordered in $<_{2}$. In Sect. 5.2 we then show how to determine minimal and maximal timestamps for each unobserved event (through a linear program) that preserves the already known ordering $<_{2}$. Inferring $<_{3}$ from the minimal (or maximal) timestamps refines $<_{2}$ and results in an SPO $L_{3}=\left(E_{2},<_{3}\right)$ which is a complete log of $M$ and has $L_{1}$ as a partial log. We explain our approach using another (more compact running) example shown in Fig. 5(a) for two bags 53 and 54 processed in the system of Fig. 3. The events in grey italic (i.e., f3, f5, f6, f14) are unobserved. 


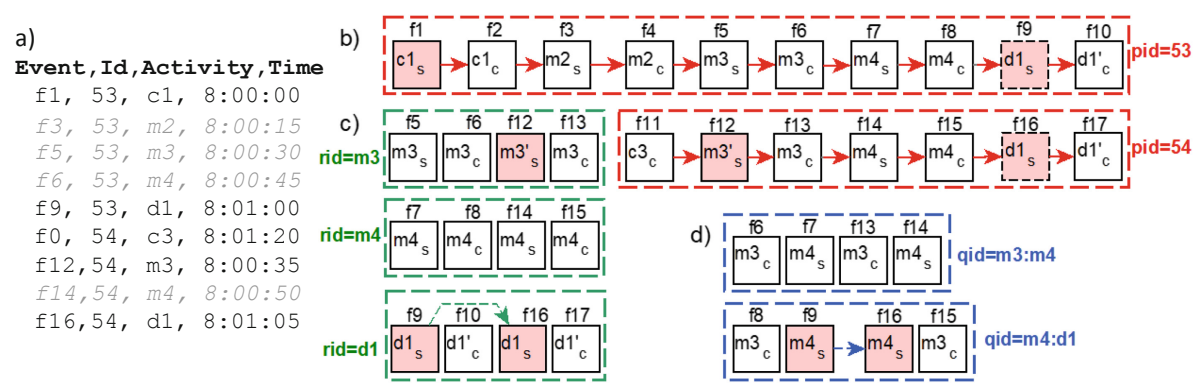

Fig. 5. Another partial event log of the system in Fig. 3 for bags 53 and 54 (a), partially complete traces of the Process (b), Resource (c) and Queue (d) proclets, restored by oracles $O_{1}, O_{2}$. Only observed events are ordered, e.g., $f 9<_{\text {rid }}^{d 1} f 16$, while the other events are isolated. (Color figure online)

\subsection{Infer Potential Complete Runs from a Partial Run}

We first derive for the partial $\log L_{1}=\left(E_{1},<_{1}\right)$ an intermediate $\log L_{2}=\left(E_{2},<_{2}\right)$ so that each trace $L_{2, p i d}^{z}$ of a process case $z$ is complete (i.e., fits the process proclet in $M$ ). In a second step, we relate each unobserved event $e \in E_{u}=E_{2} \backslash E_{1}$ to a corresponding resource and/or queue case identifier which orders observed events wrt. $<_{\text {rid }}$ and $<_{\text {qid }}$, resulting in an SPO $\pi=\left(E_{\pi},<_{\pi}\right)$ (with $E_{\pi}=E_{2}$ ). All unobserved events $e \in E_{u}$ lack a timestamp and hence are left unordered wrt. $<_{\text {rid }}$ and $<_{\text {qid }}$ in $\pi$; we later refine $<_{\pi}$ in Sect. 5.2.

We specify how to solve each of the steps in terms of two oracles $O_{1}$ and $O_{2}$ and describe concrete implementations for either. Oracle $O_{1}$ has to return $L_{2}=\left(E_{2},<_{2}\right)$ $=O_{1}\left(E_{1},<_{1}, M\right)$ by completing each partial trace $L_{1, p i d}^{z}$ of some process case $z$ into a complete trace $L_{2, \text { pid }}^{z}$ that fits the process proclet $M$. The restored unobserved events $E_{u}=E_{1} \backslash E_{1}$ only have attributes act, pid and lt (life-cycle transition) and events are totally ordered along pid, i.e., $<_{2}=<_{\text {pid }}$ is a total order. $O_{1}$ can be implemented using well-known trace alignment [3]. For example, applying $O_{1}$ on the partial log of Fig. 5(a) results in the complete process traces of Fig. 5(b). Note that, slightly deviating from our model, $O_{1}$ constructs $<_{2}$ explicitly (not based on timestamps).

Oracle $O_{2}$ has to enrich events in $L_{2}$ with information about queues and resources so that for each $e \in E_{2}$ if resource $r$ is involved in the step $\#_{a c t}(e)$, then $\#_{r i d}(e)=r$ and if queue $q$ was involved, then $\#_{q i d}(e)=q$. Moreover, in order to formulate the linear program to derive timestamps in a uniform way, each event $e$ has to be annotated with the performance information of the involved resource and/or queue. That is, if $e$ is a start event and $\#_{r i d}(e)=r \neq \perp$, then $\#_{t s r}(e)$ and $\#_{t w r}(e)$ hold the minimum service and waiting time of $r$, and if $\#_{\text {qid }}(e)=q \neq \perp$, then $\#_{t w q}(e)$ hold the minimum waiting time of $q$. For the concrete $\mathrm{PQR}$ systems considered in this paper, we set $\#_{r i d}(e)=r$ based on the model $M$ if $r$ is the case id of the resource proclet that synchronizes with transition $t=\#_{a c t}(e)$ via a channel (there is at most one). Attributes $\#_{t s r}(e), \#_{t w r}(e)$, can be set from the model as they are parameters of the resource proclet. To ease the LP formulation, if $e$ is unrelated to a resource, we set $\#_{r i d}(e)=r^{*}$ to fresh identifier and $\#_{t s r}(e)=\#_{t w r}(e)=0$; $\#_{q i d}(e)$ and $\#_{t w q}(e)$ are set correspondingly. By annotating the events in $E_{2}$, we obtain 
SPO $\pi=\left(E_{\pi},<_{\pi}\right), E_{\pi}=E_{2}$ that also contains sub-runs for each queue and resource containing all events to be complete wrt. $M$ but only observed events are ordered (due to their timestamps). For example, Fig. 5(d) shows the sub-run $\pi_{q i d}^{m 4: d l}$ containing events $f_{8}, f_{9}, f_{16}, f_{15}$ with only $f_{9}<_{\text {qid }} f_{16}$. Next, we define constraints based on the information in this intermediate $\log \pi$ to infer timestamps for all unobserved events.

\subsection{Restoring Timestamps of Unobserved Events by Linear Programming}

The SPO $\pi=(E,<)$ obtained in Sect. 5.1 from partial $\log L_{1}=\left(E_{1},<_{1}\right)$ includes all unobserved events $E_{u}=E \backslash E_{1}$ of the complete log, but lacks timestamps for each $e \in E_{u}$, \#time $(e)=\perp$. Each observed $e \in E_{1}$ has a timestamp $\#_{\text {time }}(e)$ and we also added minimum service time $\#_{t s r}$, waiting time $\#_{t w r}(e)$ of the resource $\#_{r i d}(e)$ involved in $e$ and minimum waiting time $\#_{t w q}(e)$ of the queue involved in $e$. We now define a constraint satisfaction problem that specifies the earliest $\#_{\text {tmin }}(e)$ and latest $\#_{\text {tmax }}(e)$ timestamps for each $e \in E_{u}$ so that all earliest (latest) timestamps yield a consistent ordering of all events in $E$ wrt. $<_{\text {pid }}$ (events follow the process), $<_{\text {rid }}$ (events follow resource life-cycle), and $<_{\text {qid }}$ (events satisfy queueing behavior). The problem formulation propagates the known $\#_{\text {time }}(e)$ values along with the different case notions $<_{\text {pid }},<_{\text {rid }},<_{\text {qid }}$, using $t s r, t w r, t w q$. For that, we introduce variables $x_{e}^{\mathrm{tmin}}, x_{e}^{\mathrm{tmax}} \geq 0$ for representing event attributes tmin,tmax of each $e \in E_{u}$. For all observed events $e \in E_{1}$, we set $x_{e}^{\mathrm{tmin}}=x_{e}^{\mathrm{tmax}}=\#_{\text {time }}(e)$ as here the correct timestamp is known. We now define two groups of constraints to constrain the $x_{e}^{\mathrm{tmin}}$ and $x_{e}^{\mathrm{tmax}}$ values for the unobserved events further. In the following, we assume for the sake of simpler constraints presented in this paper, that all observed events are start events (which is in line with logging in an MHS). The constraints can easily be reformulated to assume only complete events were observed (as in most business process event logs) or a mix (requiring further case distinctions).

The first group propagates constraints for $\#_{\text {time }}(e)$ along $<_{\text {pid }}$, i.e., for each token trajectory (viz. trace) $\pi_{\text {pid }}^{z}$ of pid in $\pi$. By the steps in Sect. 5.1, events in $\pi_{\text {pid }}^{z}$ are totally ordered and we write $\pi_{\text {pid }}^{z}=\left\langle e_{1} \ldots e_{m}\right\rangle$ as a sequence of events. Each process step has a start and a complete event in $\pi_{\text {pid }}^{z}$, i.e., $m=2 \cdot y, y \in \mathbb{N}$, odd events are start events and even events are complete events. For each process step $1 \leq i \leq y$, the time between start event $e_{2 i-1}$ and complete event $e_{2 i}$ is at least the service time of the resource involved (which we stored as $\#_{t s r}\left(e_{2 i-1}\right)$ in Sect. 5.1). Thus the following constraints must hold for the earliest and latest time of $e_{2 i-1}$ and $e_{2 i}$.

$$
\begin{aligned}
& x_{e_{2 i}}^{\mathrm{tmin}}=x_{e_{2 i-1}}^{\mathrm{tmin}}+\#_{t s r}\left(e_{2 i-1}\right), \\
& x_{e_{2 i}}^{\mathrm{tmax}}=x_{e_{2 i-1}}^{\mathrm{tmax}}+\#_{t s r}\left(e_{2 i-1}\right) .
\end{aligned}
$$

For the remainder, it suffices to formulate constraints only for start events. We make sure that tmin and tmax define a proper interval for each start event:

$$
x_{e_{2 i-1}}^{\mathrm{tmin}} \leq x_{e_{2 i-1}}^{\mathrm{tmax}} .
$$

We write $e_{i}^{s}=e_{2 i-1}$ for the start event of the i-th process step in $\pi_{\text {pid }}^{z}$ and $\theta_{\text {pid }}^{z}=$ $\left\langle e_{1}^{s}, \ldots, e_{m}^{s}\right\rangle$ for the sub-trace of start events of $\pi_{p i d}^{z}$. Any event $e_{i}^{s} \in \theta_{\text {pid }}^{z}$ that was 
observed in $L_{1}$, i.e., $e_{i}^{s} \in E_{1}$, has $\#_{\text {time }}\left(e_{i}^{s}\right) \neq \perp$ defined. By the assumption in Sect. 4 , $\pi_{\text {pid }}^{z}$ as well as $\theta_{\text {pid }}^{z}$ always start and end with observed events, i.e., $e_{1}^{s}, e_{y}^{s} \in E_{1}$ and $\#_{\text {time }}\left(e_{1}^{s}\right)$, \# time $_{\text {e }}\left(e_{y}^{s}\right) \neq \perp$. An unobserved event $e_{i}^{s}$ has no timestamp $\#_{\text {time }}\left(e_{i}^{s}\right)=\perp$ yet, but $\#_{\text {time }}\left(e_{i}^{s}\right)$ is bounded by $\#_{\text {time }}\left(e_{1}^{s}\right)$ (minimally) and $\#_{\text {time }}\left(e_{y}^{s}\right)$ (maximally). Furthermore, any two succeeding start events in $\theta_{\text {pid }}^{z}=\left\langle\ldots, e_{i-1}^{s}, e_{i}^{s}, \ldots\right\rangle$ are separated by the service time $\#_{t s r}\left(e_{i-1}^{s}\right)$ of step $e_{i-1}^{s}$ and the waiting time $\#_{t w q}\left(e_{i}\right)$ of the queue from $e_{i-1}$ to $e_{i}$. Similar to Eq. 1 and 2, we formulate this constraint for both $x_{e}^{\text {tmin }}$ and $x_{e}^{\text {tmax }}$ variables:

$$
\begin{aligned}
& x_{e_{k}^{s}}^{\mathrm{tmin}} \geq x_{e_{k-1}^{s}}^{\mathrm{tmin}}+\left(\#_{t s r}\left(e_{k-1}^{s}\right)+\#_{t w q}\left(e_{k}^{s}\right)\right), \\
& x_{e_{k}^{s}}^{\mathrm{tmax}} \leq x_{e_{k+1}^{s}}^{\mathrm{tmax}}-\left(\#_{t s r}\left(e_{k}^{s}\right)+\#_{t w q}\left(e_{k+1}^{s}\right)\right) .
\end{aligned}
$$

Figure 6 uses the Performance Spectrum [10] to illustrate the effect of applying our approach step by step to the partially complete traces of Fig. 5 obtained in the steps of Sect. 5.1. The straight lines in Fig. 6(a) from $f_{1}$ to $f_{9}$ (for pid=53) and from $f_{12}$ to $f_{16}$ (for pid $\left.=54\right)$ illustrate that $L_{2}\left(\right.$ after applying $\left.O_{1}\right)$ contains all intermediate steps that both process cases passed through but not their timestamps. Further (after applying $\mathrm{O}_{2}$ ), we know for each process step the resources (i.e., c1, m2, m3, m4, d1) and the queues (c1:m2, m2:m3 etc.), and their minimum service and waiting times $t s r, t w r$, twq. The sum $t s r+t w q$ is visualized as bars on the time axis in Fig. 6(a), the duration of $t w r$ is shown in Fig. 6(b). We now explain the effect of applying Eq. 4 on pid $=53$ for $f_{3}, f_{5}$ and $f_{7}$. We have $\theta_{\text {pid }}^{53}=\left\langle f_{1}, f_{3}, f_{5}, f_{7}, f_{9}\right\rangle$ with $f_{1}$ and $f_{9}$ observed, thus $x_{f_{i}}^{\text {tmin }}=$ $x_{f_{i}}^{\text {tmax }}=\#_{\text {time }}\left(f_{i}\right)$ for $i \in\{1,9\}$. By Eq. 4 , we obtain the lower-bound for the time for $f_{3}$ by $x_{f_{3}}^{\mathrm{tmin}} \geq x_{f_{1}}^{\mathrm{tmin}}+\#_{t s r}\left(f_{1}\right)+\#_{t w q}\left(f_{3}\right)$ with $\#_{t s r}\left(f_{1}\right)$ and $\#_{t w q}\left(f_{3}\right)$ the service time of resource $\mathrm{c} 1$ and waiting time of queue c1:m2. Similarly, Eq. 4 gives the lower bound for $f_{5}$ from the lower bound from $f_{3}$ etc. Conversely, the upper bounds $x_{f_{i}}^{\text {tmax }}$ are derived from $f_{9}$ "downwards" by Eq. 5. This way, we obtain for each $f_{i} \in \theta_{\text {pid }}^{53}$ an initial interval for the time of $f_{i}$ between the bounds $x_{f_{i}}^{\mathrm{tmin}} \leq x_{f_{i}}^{\mathrm{tmax}}$ as shown by the intervals in Fig. 6(a). As $x_{f_{1}}^{\mathrm{tmin}}=x_{f_{1}}^{\mathrm{tmax}}=\#_{\text {time }}\left(f_{1}\right)$ and $x_{f_{1}}^{\mathrm{tmin}}=x_{f_{1}}^{\mathrm{tmax}}=\#_{\text {time }}\left(f_{9}\right)$, the lower and upper bounds for the unobserved events in $\theta_{\text {pid }}^{53}$ form a polygon as shown in Fig. 6(b). Case 53 must have passed over the process steps and resources as a path inside this polygon, i.e., the polygon contains all admissible solutions for the timestamps of the unobserved events of $\theta_{p i d}^{53}$; we call this polygon the region of case 53. The region for case 54 overlays with the region for case 53 .

We now introduce a second group of constraints by which we infer more tight bounds for $x_{e_{i}}^{\text {tmin }}$ and $x_{e_{i}}^{\text {tmax }}$ based on the overlap with other regions. While the first group of constraints traversed token trajectories along pid (i.e., process traces), the second group of constraints traverses token trajectories for resources along rid. Each resource trace $\pi_{\text {rid }}^{r}=\left(E_{\text {rid }}^{r},<_{\text {rid }}^{r}\right)$ in $\pi$, contains all events $E_{\text {rid }}^{r}$ resource $r$ was involved in - across multiple different process traces. The SPO $<_{r i d}^{r}$ orders observed events of this resource trace due to their known timestamps; e.g. in Fig. 6(b) $f_{9}<_{\text {rid }}^{m 1} f_{16}$ with $f_{9}$ from pid $=53$ and $f_{16}$ from pid $=54$. The order of the two events $e_{p 1}^{s}<_{r i d}^{r} e_{p 2}^{s}$ for the same step $\#_{a c t}\left(e_{p 1}^{s}\right)=\#_{a c t}\left(e_{p 2}^{s}\right)=t_{1}$ in different cases $\#_{p i d}\left(e_{p 1}^{s}\right)=p 1 \neq \#_{p i d}\left(e_{p 2}^{s}\right)=p 2$ propagates "upwards" and "downwards" the process traces $\pi_{p i d}^{p 1}$ and $\pi_{p i d}^{p 2}$ as follows. Let events $f_{p 1}^{s} \in E_{p i d}^{p 1}$ and $f_{p 2} \in E_{p i d}^{p 2}$ be events in process traces $\pi_{p i d}^{p 1}$ and $\pi_{p i d}^{p 2}$ of the same 


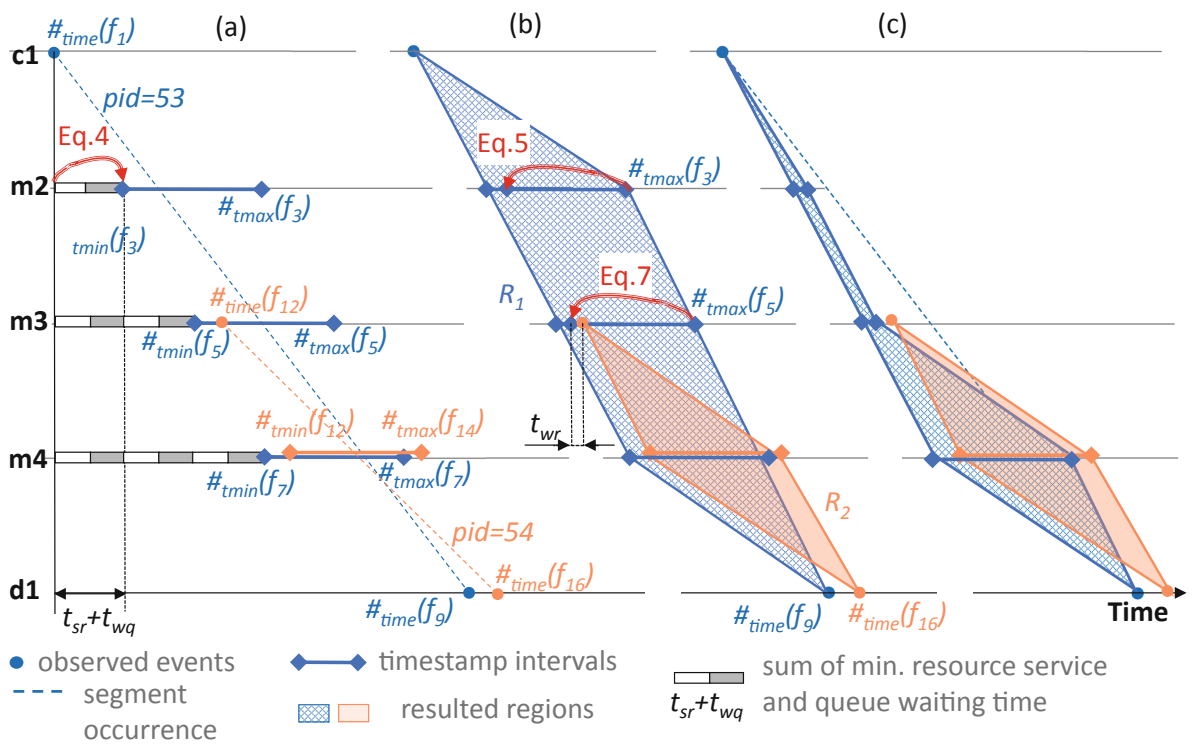

Fig. 6. Equations 1-5 define time intervals for unobserved events (a), defining regions for the possible traces (b). Equations 6-7 propagate orders of cases observed on one resource to other resources (b), resulting in tighter regions (c). (Color figure online)

step \# $\#_{a c t}\left(f_{p 1}^{s}\right)=\#_{a c t}\left(f_{p 2}^{s}\right)=t_{n}$. We say $t_{1}$ and $t_{n}$ are in FIFO relation iff there is a unique path $\left\langle t_{1} \ldots t_{n}\right\rangle$ between $t_{1}$ and $t_{n}$ in the process proclet (i.e., no loops, splits, parallelism) so that between any two consecutive transitions $t_{k}, t_{k+1}$ only synchronize with singleserver resources or FIFO queues. If $t_{1}$ and $t_{n}$ are in FIFO relation, then also $f_{p 1}^{s}<_{r i d}^{r 2} f_{p 2}^{s}$ on the resource $\mathrm{r} 2$ involved in $t_{n}$ (as the case cannot overtake the case along this path). Thus $x_{f_{p 1}^{s}}^{\mathrm{tmin}} \leq x_{f_{p 2}^{s}}^{\mathrm{tmin}}$ must hold. More specifically, $x_{f_{p 1}^{s}}^{\mathrm{tmin}}+\#_{t s r}\left(f_{p 1}^{s}\right)+\#_{t w r}\left(f_{p 1}^{s}\right) \leq x_{f_{p 2}^{s}}^{\mathrm{tmin}}$ must hold as the service time and waiting time of the resource involved in $f_{p 1}^{s}$ must elapse.

For any pair $e_{p 1}^{s}, e_{p 2}^{s} \in E_{r i d}^{r}$ with $e_{p 1}^{s}<_{r i d}^{r} e_{p 2}^{s}$ and any other trace $\theta_{r i d}^{r 2}$ for resource $r 2$ and any pair $f_{p 1}^{s}, f_{p 2}^{s} \in E_{\text {rid }}^{r 2}$ such that $\#_{p i d}\left(e_{p 1}^{s}\right)=\#_{p i d}\left(f_{p 1}^{s}\right), \#_{p i d}\left(e_{p 2}^{s}\right)=\#_{p i d}\left(f_{p 2}^{s}\right)$ and transition $\#_{a c t} e_{p 1}^{s}$ is in FIFO relation with $\#_{a c t}\left(f_{p 1}^{s}\right)$, we generate the following constraint for tmin:

$$
x_{f_{p 1}^{s}}^{\mathrm{tmin}} \leq x_{f_{p 2}^{s}}^{\mathrm{tmin}}-\left(\#_{t s r}\left(f_{p 1}^{s}\right)+\#_{t w r}\left(f_{p 1}^{s}\right)\right)
$$

and the following constraint for tmax:

$$
x_{f_{p 1}^{s}}^{\mathrm{tmax}} \leq x_{f_{p 2}^{s}}^{\mathrm{tmax}}-\left(\#_{t s r}\left(f_{p 1}^{s}\right)+\#_{t w r}\left(f_{p 1}^{s}\right)\right),
$$

In the example of Fig. 6(b), we observe $f_{9}<_{r i d}^{d 1} f_{16}$ (both of transition $d 1_{s}$ ) along resource $d 1$ at the bottom of Fig. 6(b). By Fig. $3, d 1_{s}$ and $m 3_{s}$ are in FIFO-relation. Applying Eq. 7 yields $x_{f_{5}}^{\text {tmax }} \leq \#_{\text {time }}\left(f_{12}\right)-\left(\#_{t s r}\left(f_{5}\right)+\#_{t w r}\left(f_{5}\right)\right)$, i.e., $f_{5}$ occurs at latest before $f_{12}$ minus the service and waiting time of $m 3$. This operation significantly reduces the initial region $R_{1}$. By Eq. 5 , the tighter upper bound for $f_{5}$ also propagates 
along the trace pid $=53$ to $f_{3}$, i.e., $x_{f_{3}}^{\mathrm{tmax}} \leq x_{f_{5}}^{\mathrm{tmax}}-\left(\#_{t s r}\left(f_{3}\right)+\#_{t w q}\left(f_{5}\right)\right)$, resulting in a tighter region as shown in Fig. 6(c). If another trace $\left\langle m 3_{s}, d 1_{s}\right\rangle$ were present before trace 53, then this would cause reducing the tmin attributes of the events of trace 53 by Eq. 4,6 in a similar way. In general, the more cases interact through shared resources, the more accurate timestamp intervals can be restored by Eq. 1-7 as we will show in Sect. 6.

To construct the linear program, we generate Eqs. 1 to 5 by iteration of each process trace in $L_{2}$. Further, iterate over each resource trace and for each pair of events $e_{p 1}<_{r i d}^{r}$ $e_{p 2}$ we generate Eqs. 6, 7 for each other pair of events $f_{p 1}<_{r i d}^{r 2} f_{p 2}$ that is in FIFO relation. The objective function to maximize is the sum of all intervals $\sum_{e \in E_{2}}\left(x_{e}^{\mathrm{tmax}}-\right.$ $\left.x_{e}^{\mathrm{tmin}}\right)$ to maximize the coverage of possible timestamp values by those intervals.

\section{Evaluation}

To evaluate our approach, we formulated the following questions. (Q1) Can timestamps be estimated in real-life settings and used to estimate performance reliably? (Q2) How accurately can the load (items per minute) be estimated for different system parts, using restored timestamps? (Q3) What is the impact of sudden deviations from the minimum service/waiting times, e.g., the unavailability of resource or stop/restart of an MHS conveyor, on the accuracy of restored timestamps and the computed load? For that, we extended the interactive ProM plug-in "Performance Spectrum Miner" with an implementation of our approach that solves the constraints using heuristics ${ }^{1}$. As input we considered the process of a part of real-life BHS shown in Fig. 7 and used Synthetic Logs (SL) (simulated from a model to obtain ground-truth timestamps) and Real-life Logs (RL) from a major European airport. Regarding Q3, we generated SL with regular performance and with blockages of belts (i.e., a temporary stand-still); the RL contained both performance characteristics. All logs were partial as described in Sect. 4. We selected the acyclic fragment highlighted in Fig. 7 for restoring timestamps of steps $c_{1-4}, d_{1-2}, f, s$.

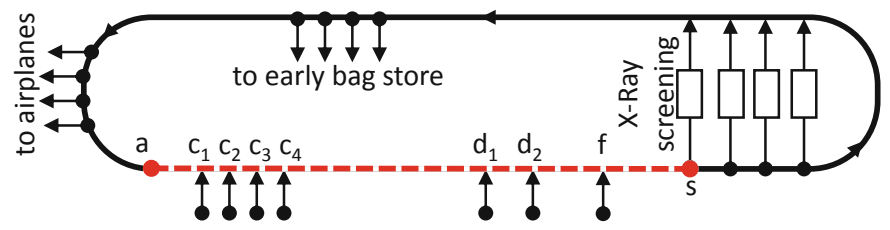

Fig. 7. In the BHS bags come from check-in counters $c_{1-4}$ and another terminals $d_{1-2}, f$, go through mandatory screening and continue to other locations. (Color figure online)

We evaluated our technique against the ground truth known for SL as follows. For each event we measured the error of the estimated timestamp intervals $\left[t_{\text {min }}, t_{\text {max }}\right]$ against the actual time $t$ as $\max \left\{\left|t_{\max }-t\right|,\left|t_{\min }-t\right|\right\}$ normalized over the sum of minimal service

\footnotetext{
${ }^{1}$ The simulation model, simulation logs, ProM plugin, and high-resolution figures are available on https://github.com/processmining-in-logistics/psm/tree/rel.
} 
and waiting times of all involved steps (to make errors comparable). We report the Mean Absolute Error (MAE) and Root Mean Square Error (RMSE) of these errors. Applying our technique to SL with regular behavior, we observed very narrow time intervals for the estimated timestamps, shown in Fig. 8(a), and a MAE of $<5 \%$. The MAE of the estimated load (computed on estimated timestamps), shown in Fig. 8(e), was $<2 \%$. For SL with blockage behavior, the intervals grew proportionally with the duration of blockages (Fig. 8(b)), leading to a proportional growth of the MAE for the timestamps. However, the MAE of the estimated load (Fig. 8(f)) was at most 4\%. The load MAE for different processing steps for both scenarios are shown in Table 1. Notably, both observed and reconstructed load showed load peaks each time the conveyor belt starts moving again.

When evaluating on the real-life event log, we measured errors of timestamps estimation as the length of the estimated intervals (normalized over the sum of minimal service and waiting times of all involved steps). Performance spectra built using the restored RL logs are shown in Fig. 8(c,d), and the load computed using these logs is shown in Fig. 8(g,h). The observed MAE was $<5 \%$ in regular behavior and increased proportionally as observed on SL. The load error could not be measured, but similarly to synthetic data, it showed peaks after assumed conveyor stops.

The obtained results on SL show that the timestamps can be always estimated, and the actual timestamps are always within the timestamp intervals (Q1). When the system resources and queues operate close to the known performance parameters $t s r, t w r, t w q$, our approach restores accurate timestamps resulting in reliable load estimates in SL (Q2). During deviations in resource performance, the errors increase proportionally with performance deviation while the estimated load remains reliable (error $<4 \%$ in SL) and shows known characteristics from real-life systems on SL and RL (Q3).
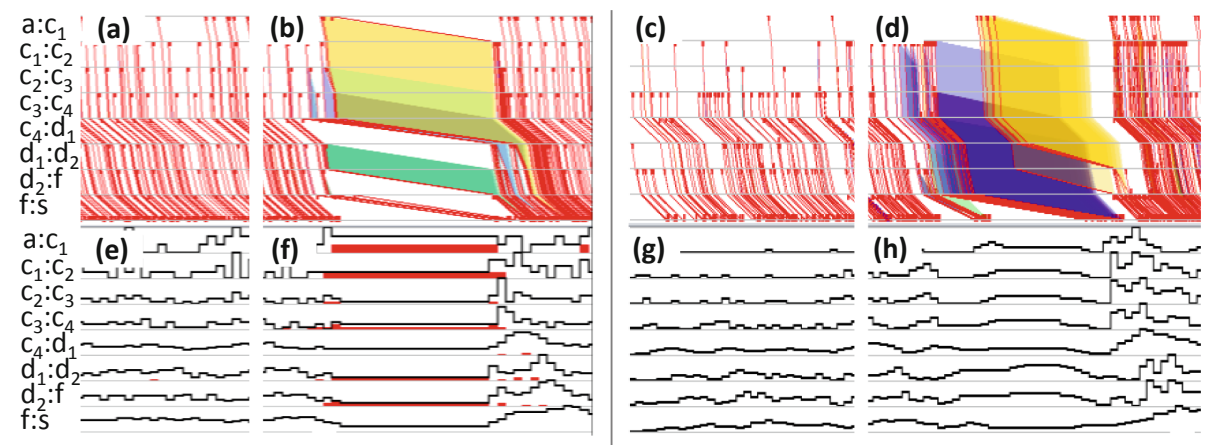

Fig. 8. Restored Performance Spectrum for synthetic $(a, b)$ and real-life $(c, d) \operatorname{logs}$. The estimated load (computed on estimated timestamps) for synthetic (e,f) and real-life (g,h) logs. For the synthetic logs, the load error is measured and shown in red (e,f). (Color figure online) 
Table 1. The estimated load (computed on estimated timestamps) Root Mean Squared Error (RMSE) and Mean Absolute Error (MAE) are shown in \% of max. load.

\begin{tabular}{l|l|l|l|l|l|l}
\hline Scenario & MAE, $c_{4}: d_{1}$ & RMSE, $c_{4}: d_{1}$ & MAE, $d_{1}: d_{2}$ & RMSE, $d_{1}: d_{2}$ & MAE, $f: s$ & RMSE, $f: s$ \\
\hline No blockages & 0.16 & 1.01 & 0.22 & 1.66 & 0.17 & 0.89 \\
Blockages & 1.67 & 4.8 & 3.19 & 7.17 & 0.15 & 0.75 \\
\hline
\end{tabular}

\section{Conclusion}

In this paper, we studied the problem of repairing a partial event log with missing events for the performance analysis of systems where case interact and compete for shared limited resources. We addressed the problem of repairing partial event logs that contain only a subset of events which impede the performance analysis of systems with shared limited resources and queues. To study and solve the problem, we used synchronous proclets [11] to model processes served by resources and queues (a PQR system). The model allows to decompose the interactions of resources and queues over multiple process cases into token trajectories for process cases, resources and queues that synchronize on shared events. We exploit the decomposition when restoring missing events along the process token trajectories using trace alignment [7]. We exploit the synchronization when formulating linear programming constraints over timestamps of restored events along, both, the process and the resource token trajectories. As a result, we obtain timestamps which are consistent for all events along the process, resource, and queue dimensions. The evaluation of our implementation in synthetic and real-life data shows errors of the estimated timestamps and of derived performance characteristics (i.e., load) of $<5 \%$ under regular performance, while correctly restoring real-life dynamics (i.e. load peaks) after irregular performance behavior.

Limitations. The work made several limiting assumptions. (1) Although the proclet formalism allows for arbitrary, dynamic synchronizations between process steps, resources, and queues, we limited ourselves in this work to a static known resource/queue id per process step. The limitation is not severe for some use cases such as analyzing MHS, but generalizing oracle $\mathrm{O}_{2}$ to a dynamic setting is an open problem. (2) The LP constraints to restore timestamps assume an acyclic process proclet without concurrency. Further, the LP constraints assume 1:1 interactions (at most one resource and/or queue per process step). Both assumptions do not hold in business processes in general; formulating the constraints for a more general setting is an open problem. (3) Our approach ensures consistency of either all earliest or all latest timestamps with the given model, it does not suggest how to select timestamps between the latest and earliest such that the consistency holds. (4) When the system performance significantly changes, e.g., due to sudden unavailability of resources, the error of restored timestamps is growing proportionally the duration of deviations. Points (3) and (4) require attention to further improve event log quality for performance analysis.

Acknowledgements. The research leading to these results has received funding from Vanderlande Industries in the project "Process Mining in Logistics". We also thank Mitchel Brunings for his comments that greatly improved our approach. 


\section{References}

1. van der Aalst, W.M.P., Barthelmess, P., Ellis, C.A., Wainer, J.: Proclets: a framework for lightweight interacting workflow processes. Int. J. Coop. Inf. Syst. 10(04), 443-481 (2001). https://doi.org/10.1142/S0218843001000412

2. van der Aalst, W.M.P.: Process Mining - Data Science in Action, 2nd edn. Springer, Heidelberg (2016). https://doi.org/10.1007/978-3-662-49851-4

3. Aalst, W.M.P., Adriansyah, A., Dongen, B.: Replaying history on process models for conformance checking and performance analysis. WIREs Data Min. Knowl. Discov. 2, 182-192 (2012). https://doi.org/10.1002/widm.1045

4. Ahmed, T., Pedersen, T.B., Calders, T., Lu, H.: Online risk prediction for indoor moving objects. In: 2016 17th IEEE International Conference on Mobile Data Management (MDM), vol. 1, pp. 102-111, June 2016. https://doi.org/10.1109/MDM.2016.27

5. Senderovich, A., Francescomarino, C.D., Maggi, F.M.: From knowledge-driven to datadriven inter-case feature encoding in predictive process monitoring. Inf. Syst. 84, 255-264 (2019). https://doi.org/10.1016/j.is.2019.01.007

6. Brown, L., et al.: Statistical analysis of a telephone call center. J. Am. Stat. Assoc. 100(469), 36-50 (2005). https://doi.org/10.1198/016214504000001808

7. Carmona, J., van Dongen, B., Solti, A., Weidlich, M.: Conformance Checking - Relating Processes and Models. Springer, Heidelberg (2018). https://doi.org/10.1007/978-3-319-994147

8. Conforti, R., La Rosa, M., ter Hofstede, A.: Timestamp repair for business process event logs. Technical report (2018/04/05 2018). http://hdl.handle.net/11343/209011

9. Denisov, V., Fahland, D., van der Aalst, W.M.P.: Predictive performance monitoring of material handling systems using the performance spectrum. In: 2019 International Conference on Process Mining (ICPM), pp. 137-144, June 2019. https://doi.org/10.1109/ICPM.2019.00029

10. Denisov, V., Fahland, D., van der Aalst, W.M.P.: Unbiased, fine-grained description of processes performance from event data. In: Weske, M., Montali, M., Weber, I., vom Brocke, J. (eds.) BPM 2018. LNCS, vol. 11080, pp. 139-157. Springer, Cham (2018). https://doi.org/ 10.1007/978-3-319-98648-7_9

11. Fahland, D.: Describing behavior of processes with many-to-many interactions. In: Donatelli, S., Haar, S. (eds.) PETRI NETS 2019. LNCS, vol. 11522, pp. 3-24. Springer, Cham (2019). https://doi.org/10.1007/978-3-030-21571-2_1

12. Gans, N., Koole, G., Mandelbaum, A.: Telephone call centers: tutorial, review, and research prospects. Manuf. Serv. Oper. Manag. 5, 79-141 (2003)

13. Lu, X., Fahland, D., van der Aalst, W.M.P.: Conformance checking based on partially ordered event data. In: Fournier, F., Mendling, J. (eds.) BPM 2014. LNBIP, vol. 202, pp. 75-88. Springer, Cham (2015). https://doi.org/10.1007/978-3-319-15895-2_7

14. Márquez-Chamorro, A.E., Resinas, M., Ruiz-Cortés, A.: Predictive monitoring of business processes: a survey. IEEE Trans. Serv. Comput. 11(6), 962-977 (2018). https://doi.org/10. 1109/TSC.2017.2772256

15. Martin, N., Depaire, B., Caris, A., Schepers, D.: Retrieving the resource availability calendars of a process from an event log. Inf. Syst. 88, 101463 (2020). https://doi.org/10.1016/j. is.2019.101463. http://www.sciencedirect.com/science/article/pii/S0306437919305150

16. Maruster, L., van Beest, N.R.T.P.: Redesigning business processes: a methodology based on simulation and process mining techniques. Knowl. Inf. Syst. 21(3), 267-297 (2009). https:// doi.org/10.1007/s10115-009-0224-0

17. Pegoraro, M., Aalst, W.: Mining uncertain event data in process mining, pp. 89-96 (2019). https://doi.org/10.1109/ICPM.2019.00023 
18. Pegoraro, M., Uysal, M.S., van der Aalst, W.M.P.: Discovering process models from uncertain event data. In: Di Francescomarino, C., Dijkman, R., Zdun, U. (eds.) BPM 2019. LNBIP, vol. 362, pp. 238-249. Springer, Cham (2019). https://doi.org/10.1007/978-3-030-37453$2 \_20$

19. Schrijver, A.: Theory of Linear and Integer Programming. Wiley, Chichester (1986)

20. Senderovich, A., Beck, J., Gal, A., Weidlich, M.: Congestion graphs for automated time predictions. In: Proceedings of the AAAI Conference on Artificial Intelligence vol. 33, pp. 4854-4861 (2019). https://doi.org/10.1609/aaai.v33i01.33014854

21. Senderovich, A., Weidlich, M., Gal, A., Mandelbaum, A.: Queue mining - predicting delays in service processes. In: Jarke, M., Mylopoulos, J., Quix, C., Rolland, C., Manolopoulos, Y., Mouratidis, H., Horkoff, J. (eds.) CAiSE 2014. LNCS, vol. 8484, pp. 42-57. Springer, Cham (2014). https://doi.org/10.1007/978-3-319-07881-6_4

22. Suriadi, S., Andrews, R., ter Hofstede, A., Wynn, M.: Event log imperfection patterns for process mining: towards a systematic approach to cleaning event logs. Inf. Syst. 64, 132-150 (2017). https://doi.org/10.1016/j.is.2016.07.011. http://www.sciencedirect.com/science/article/pii/S0306437915301344 